\title{
Musto, Marcello. L'ultimo Marx (1881-1883). Saggio di biografa intellettuale. Roma, Donzelli, 2016, 148 pp.
}

\author{
Marcelo Starcenbaum \\ Instituto de Investigación en Humanidades y Ciencias Sociales - \\ Universidad Nacional de La Plata-CONICET, Argentina \\ mstarcenbaum@gmail.com
}

Desde hace ya algunos años, las investigaciones del italiano Marcello Musto vienen produciendo aportes significativos al campo de los estudios sobre Marx y sobre el marxismo. Ya sea recuperando materiales relegados o propiciando nuevas miradas sobre problemas ya transitados, estas investigaciones constituyen uno de los pilares sobre los que se sostienen las relecturas actuales de la obra de Marx y de la cultura marxista contemporánea. En el marco de este trabajo, su libro L'ultimo Marx ha realizado un aporte específico en relación al conocimiento de la trayectoria intelectual y política del pensador alemán. Se trata de una investigación que tiene como objeto reconstruir los dos últimos años de vida de Marx. A través de la colocación de cuestiones poco atendidas y la revisión de interpretaciones ya realizadas, el libro da cuenta de una coyuntura puntual de la trayectoria de Marx en la que se condensaron grandes problemas teóricos y políticos. En tanto indagación de una biografía intelectual, el libro se detiene tanto en la dinámica de la producción teórica y la intervención política como en las cuestiones relativas a la vida personal y familiar. Lejos de constituir información meramente accesoria, las peripecias personales y familiares de Marx son analizadas de un modo que permite dar cuenta de lo estrechamente vinculada que estuvieron con sus actividades públicas como intelectual y dirigente.

El repaso biográfico propuesto en el libro está articulado sobre cuatro grandes núcleos problemáticos. El primero de ellos remite a las condiciones de vida de Marx en su estadía londinense y a la apertura a nuevos campos de investigación. Sobre el primero de los puntos, Musto da cuenta de la cotidianeidad del trabajo desarrollado en la casa situada en el número 41 de Maitland Park Road. La composición de la biblioteca, la disposición de los objetos en el estudio, la rutina de la familia y los vínculos con el exterior forman parte de una detallada reconstrucción de las condiciones bajo las cuales Marx desplegó su trabajo intelectual. Se trata de una interesante recolocación de Marx en la que tienen lugar las dimensiones político-intelectuales de su biografía, como el seguimiento de los sucesos internacionales y el diálogo mantenido en su hogar con dirigentes e intelectuales que viajaban especialmente a Londres para consultarlo, pero también las personales, como sus deterioradas condiciones de salud y la tristeza por el regreso de sus nietos a Francia. Sobre el segundo de los aspectos mencionados, Musto analiza el desplazamiento del interés de Marx hacia la antropología y las matemáticas. La lectura de Morgan y otros antropólogos contemporáneos es enmarcada en el objetivo de profundizar el conocimiento de períodos y áreas fundamentales para la continuidad del proyecto de crítica de la economía política y de incorporar una información más detallada que aquella con la que había contado en las décadas de 1850 y 1860. Asimismo, Musto recorre los Cuadernos etnológicos a los fines de constatar la no influencia del evolucionismo que caracterizaba a las obras que habían sido objeto de comentarios y refrendar la idea de que en Marx se encuentra una concepción compleja y multilineal de la historia. Con respecto a las matemáticas, se destaca el pasaje desde una concepción de la disciplina como subsidiaria del

Recepción: 17 de julio de 2019 | Aprobación: 15 de agosto de 2019 | Publicación: 15 de enero de 2020

Cita sugerida: Starcenbaum, M. (2020). [Revisión del libro L’ultimo Marx (1881-1883). Saggio di biografia intellettuale por M. Musto]. Trabajos y Comunicaciones (51), e114. https://doi.org/10.24215/23468971e114 
estudio económico hacia una valoración específica del trabajo científico sobre los números. De este modo, se repasan los Manuscritos matemáticos con el objetivo de evidenciar el interés por las matemáticas puras y la importancia que adquirió esta ciencia en momentos de gran dificultad personal.

El segundo está relacionado con los debates alrededor del desarrollo del capitalismo en Rusia. En primer lugar, Musto interroga la producción de los últimos años de Marx a partir de la idea del capitalismo como presupuesto necesario de la sociedad comunista. En sintonía con otras lecturas de Marx que destacaron un desplazamiento en la interpretación del lugar de Rusia en la futura revolución proletaria, este análisis da cuenta del pasaje desde una concepción del país oriental como baluarte contrarrevolucionario hacia la percepción de que allí existían un conjunto de condiciones favorables para una revolución social. En este sentido, Musto regresa al ya célebre intercambio con Vera Zasúlich para certificar que Marx jamás afirmó que la sociedad humana estaba destinada a transitar el mismo recorrido histórico. Del mismo modo, recupera la crítica de Marx a las interpretaciones de Nikolai Mijailovski en términos de una anticipación de las tendencias del marxismo contemporáneo a convertir la obra de Marx en una teoría histórico-filosófica de carácter universal. En segundo lugar, Musto vuelve al debate acerca del supuesto eurocentrismo de Marx para afirmar que su concepción acerca de la transición al capitalismo desde formas comunitarias se modificó sensiblemente a lo largo de su trayectoria. En este sentido, la lectura de los materiales de estos años evidencia que, sin romper totalmente con las nociones a través de las cuales había pensado la situación de Rusia, se produce una apertura teórica que permite considerar otras vías posibles para la transición al socialismo. Según este recorrido, el contacto establecido por Marx con los populistas rusos desempeñó un rol clave en la consolidación de una concepción multilineal de la historia y de un consecuente internacionalismo a escala global. La importancia de estos años radica por tanto en ellos aflora la idea acerca de la posibilidad de que la revolución se produzca en condiciones y formas hasta entonces jamás vislumbradas.

El tercero combina, al igual que el primero, un problema de índole político-intelectual y uno de tipo personal. Por un lado, Musto reconstruye el proceso de circulación de El Capital en los países europeos y la incipiente conversión de Marx en una figura de relevancia internacional. De esta manera, se establece una secuencia que arranca en 1840, en la que Marx era conocido por un grupo limitado de intelectuales y dirigentes, continúa con el desarrollo de la Primera Internacional y la Comuna de París, los cuales contribuyen a una mayor notoriedad de su figura, y se corona con la difusión de su obra magna, a partir de la cual el nombre de Marx comenzará a ser una referencia ineludible en los debates políticos e intelectuales. Lejos de toda mitificación, Musto lleva a cabo una descripción del proceso de difusión de la obra marxiana en la que se destaca el hecho de que en los diferentes países europeos las ideas de Marx debieron competir, a veces desde una posición minoritaria, con las de los otros pensadores socialistas contemporáneos. Por otra parte, se reconstruyen las peripecias familiares a partir del deterioro de la salud Jenny von Westphalen así como los momentos posteriores a su fallecimiento en diciembre de 1881 . Al respecto, Musto recupera la ayuda brindada por Engels en aquella coyuntura crítica y la especificidad de las tareas intelectuales reanudadas por Marx meses después de la muerte de su esposa. De este modo es analizada la cronología comentada realizada por Marx a partir de la lectura de un conjunto de libros históricos. Este trabajo es interpretado en términos de un esfuerzo por contrastar las bases de su concepción teórica con los procesos históricos que habían marcado la historia de la humanidad. Reforzando la idea de que no se encuentra en su obra una posición determinista, este análisis destaca el interés de Marx en el problema de la conformación histórica del Estado moderno.

Por último, Musto reconstruye los tramos finales de la vida de Marx. Los meses que van desde comienzos de 1882 a principios de 1883 son analizados a través de tres grandes hitos. Uno de ellos es el viaje a Argelia. Al respecto, el libro se detiene en las impresiones del país africano que Marx le hace llegar a sus hijas y a Engels. Al respecto se analizan los comentarios realizados por Marx sobre el sistema de propiedad de la tierra, la escasa presencia del Estado y las costumbres de la población africana. Asimismo, este recorrido da cuenta de su penosa situación de salud y los esfuerzos por curarse en un clima distinto al del norte europeo. El segundo de ellos es el accidentado regreso a Londres, que conlleva una estadía forzada en Mónaco y una serie 
de caracterizaciones de un lugar que se volvía privilegiado para la crítica social. Sobre esta permanencia en Mónaco se destaca la asistencia de Marx a la sala de lectura del Casino, la vinculación personas extranjeras que circulaban por la ciudad y la descripción mordaz de las escenas en las que jugadores de toda Europa gastaban fortunas en los juegos de ruleta. El tercero es el de sus últimos días. Aquí Musto repasa la crítica radical a la que Marx sometió a aquellos intelectuales que aún se encontraban influenciados por Proudhon y Bakunin y el esfuerzo por contrarrestar ciertos efectos de la difusión de su obra. Al respecto se presenta una interesante reconstrucción del surgimiento y los efectos de la frase "lo único que sé es que no soy marxista”. Asimismo, el autor da cuenta de los padecimientos de salud de Marx, que lo condujeron cada vez más a la reclusión de su hogar, así como de las dolencias del espíritu, especialmente luego de la muerte de su hija Jenny. Finalmente, a modo de epílogo, se reconstruyen las circunstancias que rodearon su muerte ocurrida el 14 de marzo de 1883.

Junto al análisis de estos cuatros núcleos que conforman la biografía intelectual del último Marx, el libro de Musto interviene en el espectro de interpretaciones y discusiones suscitadas a partir de los giros y transformaciones producidos en estos últimos dos años. En notas al pie ubicadas en el medio del tratamiento de los avatares personales y producciones intelectuales del período 1881-1883, Musto procede a complejizar, matizar y refutar algunas consideraciones clásicas y contemporáneas sobre la obra tardía de Marx. Algunas de ellas están relacionadas con los términos a través de los cuales se deben entenderse aspectos de su trayectoria intelectual. Por ejemplo, el libro intenta proporcionar una visión distinta de aquellos años que la que presentan algunas de las biografías más importantes de Marx. Contra Vorländer y Berlin, que dan distintas versiones de un período marcado por la pérdida de la capacidad de trabajo y una especie de "muerte lenta", Musto afirma que Marx no sólo continuó sus investigaciones sino que las expandió hacia nuevas disciplinas como la antropología y las matemáticas. Apoyándose en las biografías escritas por Mehring y Riazánov, el libro da cuenta que si bien la última fase de creación intelectual de Marx fue compleja y estuvo en algún sentido limitada, también fue muy importante desde el punto de vista teórico. En otro caso, Musto se esfuerza por reconstruir de manera precisa tanto el viaje de Marx a Argelia como los efectos de la estadía africana en sus posiciones políticas e intelectuales. En este sentido, el autor llama la atención sobre la poca importancia que se le otorga a este episodio en la vida de Marx, presentando como caso sintomático la biografía realizada por el intelectual de origen argelino Jacques Attali. Pero al mismo tiempo afirma que no deben ser totalmente refrendadas las afirmaciones de Lafargue de que Marx habría regresado de Argelia con "la cabeza llena de África y de árabes". Como queda demostrado en la reconstrucción del viaje, las condiciones de salud le impidieron a Marx abocarse al estudio sistemático de la realidad argelina pero al mismo tiempo no constituyeron un obstáculo para la curiosidad y la indagación sobre el país africano.

Otras aclaraciones tienen menos que ver con dimensiones de la trayectoria intelectual que con grandes núcleos teóricos y políticos de su obra. Por ejemplo, Musto trata de desmontar la idea de un Marx eurocéntrico y orientalista. A su entender, dicha caracterización es producto de lecturas superficiales y limitadas que trasladaron a la totalidad de la obra de Marx los rasgos de una reflexión ingenua sobre el colonialismo realizada por un joven periodista de 35 años. De este modo, el libro discute con las tesis de Said, a las cuales califica de tendenciosas, colocando en primer plano las críticas de las que fue objeto la caracterización de Marx en las páginas de Orientalismo. Así ocurre con la lectura de al-Azm, quien acusó a Said de trasladar la idea de la superioridad contingente de Europa frente a Oriente en una relación necesaria válida para todos los tiempos históricos, y la de Ahmad, quien afirmó que la incorporación de Marx al espectro orientalista sólo es posible a condición de descontextualizar las citas. Al respecto no resulta casual que, frente a la interpretación de Said, Musto reivindique el libro Marx at the Margins de Kevin Anderson, un trabajo dedicado a analizar las aproximaciones positivas de Marx a las realidades de la periferia del mundo capitalista. Sí resulta llamativo la ausencia en estas referencias de Marx y América Latina de José Aricó, un trabajo pionero en la crítica al supuesto de un Marx eurocéntrico. Lo mismo cabe decir acerca de la interpretación del desplazamiento analítico sobre la situación de la comuna rural rusa. Según Musto, lo que se produce en los borradores de la respuesta a Vera Zasúlich es la consideración de otras vías para la transición al socialismo 
pero no una transformación total de su interpretación de la comuna rural. De este modo su lectura difiere de la hipótesis del viraje radical sostenida por Shanin y Dussel así como de la idea de un "Marx tercermundista" que habría depositado las tareas revolucionarias en las masas rurales de la periferia.

Para concluir, puede afirmarse que la investigación desarrollada por Musto constituye un aporte doble al conocimiento sobre la trayectoria política e intelectual de Marx. Por un lado, se trata de una reconstrucción biográfica que se concentra en un período corto pero relevante en cuanto a los desplazamientos que allí se condensan. Frente a otros esfuerzos biográficos que atienden la totalidad de su trayectoria y análisis que se centran predominantemente entre las décadas de 1840 y 1870, el libro de Musto da cuenta de un conjunto de problemas que sólo pueden ser comprendidos en su especificidad a partir del recorte de los dos últimos años de vida de Marx. Por otra parte, la atención particularizada de estos problemas conlleva la discusión con otras lecturas de Marx y el marxismo que también han atendido las transformaciones de estos dos años o que las han relacionado con el resto de su trayectoria teórica. Enfrentada a las operaciones de subestimación o exageración de los desplazamientos operados en estos años -un Marx eurocéntrico/un Marx tercermundista, un Marx poco interesado en África/un Marx africanizado-, la reconstrucción de Musto intenta delimitar con precisión las complejos cambios producidos antes de su muerte. En fin, son estos aportes los que permiten perfilar a L'ultimo Marx como un aporte fundamental en el necesario e inacabado esfuerzo por dar cuenta de la singularidad de la trayectoria política e intelectual del pensador alemán. 